\title{
Endoscopy Timing in Patients with Acute Upper Gastrointestinal Bleeding
}

\author{
Gonçalo Alexandrino ${ }^{1}$, Tiago Dias Domingues ${ }^{2}$, Rita Carvalho ${ }^{1}$, Mariana Nuno Costa ${ }^{1}$, Luís Carvalho Lourenço ${ }^{1}$ and Jorge Reis ${ }^{1}$ \\ ${ }^{1}$ Hospital Professor Doutor Fernando Fonseca, Serviço de Gastrenterologia, Amadora, ${ }^{2}$ Centro de Estatística e Aplicações da Universidade \\ de Lisboa (CEAUL), Lisbon, Portugal
}

Background/Aims: The role of very early ( $\leq 12$ hours) endoscopy in nonvariceal upper gastrointestinal bleeding is controversial. We aimed to compare results of very early and early (12-24 hours) endoscopy in patients with upper gastrointestinal bleeding demonstrating low-risk versus high-risk features and nonvariceal versus variceal bleeding.

Methods: This retrospective study included patients with nonvariceal and variceal upper gastrointestinal bleeding. The primary outcome was a composite of inpatient death, rebleeding, or need for surgery or intensive care unit admission. Endoscopy timing was defined as very early and early. We performed the analysis in two subgroups: (1) high-risk vs. low-risk patients and (2) variceal vs. nonvariceal bleeding.

Results: A total of 102 patients were included, of whom 59.8\% underwent urgent endoscopy. Patients who underwent very early endoscopy received endoscopic therapy more frequently $(p=0.001)$, but there was no improvement in other clinical outcomes. Furthermore, patients at low risk and with nonvariceal bleeding who underwent very early endoscopy had a higher risk of the composite outcome.

Conclusions: Very early endoscopy does not seem to be associated with improved clinical outcomes and may lead to poorer outcomes in specific populations with upper gastrointestinal bleeding. The actual benefit of very early endoscopy remains controversial and should be further clarified. Clin Endosc 2019;52:47-52

Key Words: Endoscopy, gastrointestinal; Endoscopy, digestive system; Gastrointestinal hemorrhage; Hemostasis, endoscopic; Patient outcome assessment

\section{INTRODUCTION}

Acute upper gastrointestinal bleeding (UGIB) is a frequent cause of hospital admission. The initial management is based on fluid resuscitation and blood transfusion whenever indicated, followed by upper digestive endoscopy for both diagnostic and therapeutic purposes, with endoscopic hemostasis

Received: June 4, 2018 Revised: August 18, 2018

Accepted: August 27, 2018

Correspondence: Gonçalo Miguel Alves Alexandrino

Hospital Professor Doutor Fernando Fonseca, Serviço de Gastrenterologia, IC19, Amadora 2720-276, Portugal

Tel: +351-96-619-9616, Fax: +351-96-619-9616, E-mail: goncaloalexandrino@ gmail.com

ORCID: https://orcid.org/0000-0002-1931-948X

(c) This is an Open Access article distributed under the terms of the Creative Commons Attribution Non-Commercial License (http://creativecommons.org/ licenses/by-nc/3.0) which permits unrestricted non-commercial use, distribution, and reproduction in any medium, provided the original work is properly cited. being the mainstay of treatment. ${ }^{1,2}$

For nonvariceal UGIB, current guidelines recommend performing early endoscopy (within the first 24 hours of admission). Patients with high-risk features, including hemodynamic instability refractory to volume resuscitation, bloody emesis/nasogastric aspirate during hospitalization, and contraindication to discontinuation of anticoagulants should be considered candidates for very early endoscopy (within the first 12 hours of admission). ${ }^{1}$ In cases of variceal bleeding, endoscopy should be performed within the first 12 hours. ${ }^{2,3}$

Nevertheless, the role of very early endoscopy ( $\leq 12$ hours) in high-risk patients with nonvariceal bleeding is still controversial and a matter of intense debate. ${ }^{4}$ Latest research revealed that very early endoscopy leads to a higher probability of detecting high-risk lesions, warranting endoscopic intervention without improved clinical results, including need for surgery or death. ${ }^{5-7}$ However, in those studies, patients were 
not classified based on severity of bleeding, so it is unclear if the timing of endoscopy predicts clinical outcomes in highrisk patients. On the other hand, Lim et al. concluded that the high-risk population, with a Glasgow Blatchford score (GBS) $\geq 12$, benefited from very early endoscopy and had a lower mortality rate. ${ }^{8}$ The results differ from those of Kumar et al., which showed that very early endoscopy was not associated with better clinical outcomes because it was a predictor of poorer outcomes in the low-risk population and had no effect on outcomes in high-risk patients. ${ }^{9}$

Furthermore, most studies regarding the ideal timing of endoscopy in UGIB focused only on patients with nonvariceal bleeding and excluded those with variceal bleeding. However, when a patient presents with UGIB, the etiology of bleeding cannot be confirmed prior to endoscopy. Hence, in this study we included UGIB patients with variceal and nonvariceal bleeding. The importance of the timing of endoscopy in UGIB is further highlighted by the report of weekend effect, whereby the prognosis of patients presenting after hours may be worse. ${ }^{10,11}$

Our aim was to assess the relation between endoscopy timing and clinical outcomes in patients with UGIB admitted to a Portuguese emergency department and evaluate if patients with low versus high-risk features and variceal bleeding versus nonvariceal bleeding have different outcomes with very early versus early endoscopy.

\section{MATERIALS AND METHODS}

We conducted a retrospective cohort study, which included consecutive patients admitted with UGIB over a period of 10 months, between January and October 2015.

All patients underwent endoscopy in the first 24 hours since admission. Timing of endoscopy was defined as very early $(\leq 12$ hours) and early (12-24 hours) from the time of admission, according to the current guidelines. ${ }^{1}$ Endoscopy timing was considered as the interval between arrival at hospital and the beginning of examination. Very early endoscopy was available on a 24/7 basis, according to the gastroenterologist's decision. Hemostatic therapy was administered according to the most recent clinical evidence.

The primary composite outcome considered was death, bleeding recurrence, and need for surgery or intensive care unit (ICU) admission during hospital stay. Inpatient rebleeding was defined as bleeding confirmed on repeat endoscopy. Secondary outcomes were also assessed, including need for endoscopic therapy, length of stay, and need for blood transfusion. The medical files were periodically assessed to check for development of the above clinical outcomes.
Demographic and medical history data were collected. Vital signs and blood test values were recorded to assess GBS, a widely studied score that can be assessed at the time of patient admission. GBS is the recommended score in the guidelines for nonvariceal bleeding, ${ }^{1}$ and it does not require results of endoscopy; hence, it was previously used in the literature for this purpose. We used it to classify patients as those at lowrisk (GBS $<12$ ) and high-risk (GBS $\geq 12$ ).

Patient data were presented using means and standard deviations or median and interquartile range for continuous variables and counts and percentages for categorical ones. Baseline features within subgroups were assessed with $t$-tests for the continuous variables and the Pearson chi-square test for the binary variables. Univariate relations between population features and the composite outcomes were tested and presented with odds ratios (ORs) from logistic regression models. $P$-values $<0.05$ were considered significant. All analyses were evaluated using SPSS (Statistical Package for Social Science) version 22. We also performed the statistical analysis in subgroups: (1) high versus low-risk patients, based on the GBS and (2) variceal versus nonvariceal bleeding.

\section{RESULTS}

\section{Patient characteristics}

A total of 102 patients were included: $75.5 \%(n=77)$ were male and the mean age was 67 years $( \pm 14.17)$. Very early endoscopy was performed in $59.8 \%(n=61)$ and early endoscopy in $40.2 \%(n=41)$. The mean GBS was 10.1. None of the patients in the study had the need for interventional radiologic therapy. The characteristics and clinical outcomes of the patients are available in Table 1. Tables 2 and 3 summarize the characteristics and clinical outcomes of the very early vs. early endoscopy and nonvariceal vs. variceal bleeding subgroups, respectively.

\section{Patient outcomes}

At least one of the components of the primary composite outcomes was noted in 34 patients (33.3\%): death in 6.9\% $(n=7)$, rebleeding in $14.7 \%(n=15)$, need for surgery in $5.9 \%$ $(n=6)$, and ICU admission in $5.9 \%(n=6)$. All deaths occurred after the endoscopy. In terms of secondary outcomes, 50\% $(n=51)$ received endoscopic therapy, and $45.1 \%(n=46)$ received one or more blood transfusions during admission. The mean number of erythrocyte concentrate units used for each patient was $2.7( \pm 3.1)$. The mean duration of hospital permanence was 8.9 days $( \pm 5.2)$. 


\section{Predictors of composite outcome}

Variceal bleeding (OR, 8.8; 95\% confidence interval [CI], 2.43-31.872; $p<0.001$ ) and time from admission to endoscopy (OR, $0.261 ; 95 \%$ CI, $0.113-0.602$; $p=0.001$ ) were statistically significant predictors of the composite outcome, with a $73.9 \%$ lower risk of the composite outcome in cases of more time since admission to endoscopy. All other variables, including age, sex, GBS, and length of stay, were not significant predictors (Table 4).

\section{Very early versus early endoscopy}

Those who underwent very early endoscopy were more likely to receive endoscopic therapy than those who underwent early endoscopy ( $63.9 \%$ vs. $29.3 \%, p=0.001)$. We found no relation between timing of endoscopy and other clinical outcomes (Table 2).

\section{Effect of endoscopy timing according to patient's risk}

Seventy patients (68.6\%) had low risk of bleeding. Of these, 37 (52.9\%) experienced one of the primary outcomes. Variceal

Table 1. Characteristics and Clinical Outcomes of the Study Population $(n=102)$

\begin{tabular}{lc}
\hline Age (yr) & $67.18 \pm 14.17$ \\
Male sex & $77(75.5 \%)$ \\
Endoscopy timing & $61(59.8 \%)$ \\
Very early & $41(40.2 \%)$ \\
Early & \\
Bleeding etiology & $77(77.5 \%)$ \\
Nonvariceal bleeding & $25(24.5 \%)$ \\
Variceal Bleeding & \\
Patient risk & $70(68.6 \%)$ \\
Lower-risk & $32(31.4 \%)$ \\
Higher-risk & $10.1(\mathrm{minimum:} 0 ;$ \\
Glasgow-Blatchford score & maximum: 20$)$ \\
& \\
Primary outcome & $34(33.3 \%)$ \\
Composite outcome & \\
Components of the primary outcome & $7(6.9 \%)$ \\
Inpatient death & $15(14.7 \%)$ \\
Inpatient rebleeding & $6(5.9 \%)$ \\
Surgical intervention & $6(5.9 \%)$ \\
ICU admission & $51(50.0 \%)$ \\
Secondary outcomes & $46(45.1 \%)$ \\
Endoscopic intervention & $8.9 \pm 5.2$ \\
Need for blood transfusion & \\
Mean time of hospital stay (days) & \\
\hline
\end{tabular}

Data are presented as mean \pm standard deviation or number (\%). ICU, intensive care unit. bleeding (OR, 10.568; 95\% CI, 2.191-50.967; $p=0.001$ ) and time to endoscopy (OR, $0.273 ; 95 \% \mathrm{CI}, 0.100-0.743 ; p=0.01$ ) were statistically significant factors with a decreased risk of $72.7 \%$ of reaching the composite outcome with early endoscopy, and $32(31.4 \%)$ patients had high-risk features. However, there were no predictors of the composite outcome, including endoscopy timing $(p=0.130$ ). As expected, deaths during hospitalization $(p=0.004)$ and need for surgery $(p=0.011)$ were more likely to occur in high-risk patients. The results are expressed in Tables 5 and 6 .

\section{Effect of the endoscopy timing according to bleed- ing etiology}

Seventy-seven patients (75.5\%) were diagnosed with nonvariceal bleeding versus 25 (24.5\%) with variceal bleeding. Variceal and nonvariceal bleeding were more frequent in males and females, respectively $(p=0.027)$. Patients with variceal bleeding were more likely to receive endoscopic therapy $(p<0.001)$ and reach the composite outcome $(p<0.001)$. Patients with variceal bleeding had no different clinical outcomes, whether they underwent very early or early endoscopy $(p=0.231)$. However, patients with nonvariceal bleeding who underwent very early endoscopy were more likely to reach the composite endpoint (OR, 0.260; 95\% CI, 0.098-0.687; $p=0.006$ ) and require surgical intervention $(p=0.034)$. The results are summarized in Tables 7 and 8 .

\section{DISCUSSION}

In our study with 102 patients with UGIB, timing of endoscopy was revealed to be a predictor of clinical composite outcomes, including death, bleeding recurrence, and need for surgery or ICU admission. There was a 73.9\% lower chance of experiencing the composite outcome with early rather than with very early endoscopy. When we analyzed the subgroups, very early endoscopy was a significant predictor of poorer results only in the low-risk group and patients with nonvariceal bleeding.

Some studies evaluated the role of timing of endoscopy on clinical results, mainly in patients with nonvariceal bleeding. However, they had different designs. Several previous studies revealed that in patients who undergo endoscopy earlier, the possibility of detecting high-risk bleeding lesions is greater, resulting in a higher rate of endoscopic intervention, than in patients who undergo endoscopy later. However, these studies did not show an improvement in the clinical results of patients, including death, bleeding recurrence, surgery, or hospital stay. ${ }^{5-7}$ Thus, previous research did not show a clear advantage of very early endoscopy in UGIB. Two different studies 
Table 2. Characteristics and Clinical Outcomes of Patients Receiving Very Early vs. Early Endoscopy $(n=102)$

\begin{tabular}{|c|c|c|c|}
\hline & Very early & Early & $p$-value \\
\hline Number of patients & $61(59.8 \%)$ & $41(40.2 \%)$ & \\
\hline Age (yr) & $67 \pm 14$ & $73 \pm 13$ & 0.503 \\
\hline Male sex & $46(75.4 \%)$ & $31(75.6 \%)$ & 0.982 \\
\hline \multicolumn{4}{|l|}{ Bleeding etiology } \\
\hline Nonvariceal bleeding & $44(72.1 \%)$ & $33(80.5 \%)$ & \multirow{2}{*}{0.336} \\
\hline Variceal Bleeding & $17(27.9 \%)$ & $8(19.5 \%)$ & \\
\hline \multicolumn{4}{|l|}{ Clinical and laboratory characteristics } \\
\hline Any antiplatelet therapy & $16(26.2 \%)$ & $13(31.7 \%)$ & 0.655 \\
\hline Any anticoagulation & $2(3.3 \%)$ & $7(17.1 \%)$ & 0.028 \\
\hline Initial systolic blood pressure ( $\mathrm{mm} \mathrm{Hg}$ ) & $120(37)$ & $126(22)$ & 0.707 \\
\hline Initial heart rate (bpm) & $94(33)$ & $90(26)$ & 0.150 \\
\hline Shock & $29(47.5 \%)$ & $13(31.7 \%)$ & 0.151 \\
\hline Altered mental status & $10(16.4 \%)$ & $3(7.3 \%)$ & 0.233 \\
\hline Hemoglobin (g/dL) & $9.58 \pm 2.46$ & $8.82 \pm 2.78$ & 0.158 \\
\hline Platelet count $\left(\mathrm{x} 10^{3} / \mathrm{mL}\right)$ & $192(124)$ & $232(209.5)$ & 0.116 \\
\hline INR & $1.0(0.3)$ & $1.1(0.4)$ & 0.342 \\
\hline Chronic liver disease & $19(31.3 \%)$ & $9(22.0 \%)$ & 0.369 \\
\hline Chronic kidney disease & $2(3.3 \%)$ & $8(19.5 \%)$ & 0.013 \\
\hline Glasgow-Blatchford score & $10.05 \pm 4.44$ & $10.27 \pm 4.28$ & 0.805 \\
\hline \multicolumn{4}{|l|}{ Primary outcome } \\
\hline Composite outcome & $42(68.9 \%)$ & $15(36.6 \%)$ & 0.001 \\
\hline \multicolumn{4}{|l|}{ Components of the primary outcome } \\
\hline Inpatient death & $4(6.6 \%)$ & $3(7.3 \%)$ & 1.0 \\
\hline Inpatient rebleeding & $10(16.4 \%)$ & $5(12.2 \%)$ & 0.557 \\
\hline Surgical intervention & $6(9.8 \%)$ & $0(0.0 \%)$ & 0.079 \\
\hline ICU admission & $6(9.8 \%)$ & $0(0.0 \%)$ & 0.079 \\
\hline \multicolumn{4}{|l|}{ Secondary outcomes } \\
\hline Endoscopic intervention & $39(63.9 \%)$ & $12(29,3 \%)$ & 0.001 \\
\hline Need for blood transfusion & $20(32.8 \%)$ & $26(63.4 \%)$ & 0.327 \\
\hline Mean time of hospital stay (days) & $7 \pm 4.8$ & $8 \pm 5.4$ & 0.629 \\
\hline
\end{tabular}

Data are presented as mean \pm standard deviation or median (interquartile range) for continuous variables and as $n$ (\%) for categorical variables.

INR, international normalized ratio; ICU, intensive care unit.

investigated if prognostic scores could help the decision of when to perform endoscopy. ${ }^{8,9}$ These studies stratified patients into low-risk (GBS $<12$ ) and high-risk (GBS $\geq 12$ ) groups. Lim et al. concluded that in the high-risk group, endoscopy timing was the only significant predictor of death because $100 \%$ of the patients who underwent endoscopy in the first 13 hours survived, whereas there was a $44 \%$ mortality rate with later endoscopy. ${ }^{8}$

Our results are in accordance with previous data that did not reveal differences in mortality rates with very early vs. early endoscopy. Nevertheless, we saw an increased risk of a clinical composite outcome (death, rebleeding, or need for surgery or ICU admission) in patients who underwent very early endoscopy, especially those with low-risk features and nonvariceal bleeding. These results are in agreement with those of the study conducted by Kumar et al., which was the first to suggest a potential risk in performing early endoscopy. ${ }^{9}$ The authors suggested that the low-risk population who had earlier access to endoscopy were not provided adequate fluid resuscitation because they may have seemed less unstable on presentation, and they pointed out that medical therapy and not the endoscopy timing was most crucial in preventing unfavorable results in UGIB. ${ }^{9}$

Most studies in this field focus only on patients with nonvariceal bleeding, but prior to endoscopy, etiology of bleeding can only be speculated, so we included patients with variceal and nonvariceal bleeding in our study. To the best of our knowledge, we present the first research that stratified patients 
Table 3. Characteristics and Clinical Outcomes of Patients with Nonvariceal vs. Variceal Bleeding ( $n=102)$

\begin{tabular}{|c|c|c|c|}
\hline & Nonvariceal bleeding & Variceal bleeding & $p$-value \\
\hline Number of patients & $77(75.5 \%)$ & $25(24.5 \%)$ & \\
\hline Age (yr) & $69.01 \pm 14.59$ & $61.52 \pm 11.24$ & 0.021 \\
\hline Male sex & $54(70.1 \%)$ & $23(92.0 \%)$ & 0.027 \\
\hline \multicolumn{4}{|l|}{ Endoscopy timing } \\
\hline Very early & $44(57.1 \%)$ & $17(68.0 \%)$ & \multirow{2}{*}{0.336} \\
\hline Early & $33(42.9 \%)$ & $8(32.0 \%)$ & \\
\hline Glasgow-Blatchford score & $9.95 \pm 4.53$ & $10.72 \pm 3.79$ & 0.444 \\
\hline \multicolumn{4}{|l|}{ Primary outcome } \\
\hline Composite outcome & $35(45.5 \%)$ & $22(88.0 \%)$ & $<0.001$ \\
\hline \multicolumn{4}{|l|}{ Components of the primary outcome } \\
\hline Inpatient death & $5(6.5 \%)$ & $2(8.0 \%)$ & 1.0 \\
\hline Inpatient rebleeding & $9(11.7 \%)$ & $6(24.0 \%)$ & 0.19 \\
\hline Surgical intervention & $6(7.8 \%)$ & $0(0.0 \%)$ & 0.332 \\
\hline ICU admission & $3(3.9 \%)$ & $3(12.0 \%)$ & 0.156 \\
\hline \multicolumn{4}{|l|}{ Secondary outcomes } \\
\hline Endoscopic intervention & $30(39.0 \%)$ & $21(84.0 \%)$ & $<0.001$ \\
\hline Blood transfusion & $24(31.2 \%)$ & $22(88.0 \%)$ & 0.083 \\
\hline Mean time of hospital stay (days) & $7 \pm 3.9$ & $9 \pm 5.4$ & 0.047 \\
\hline
\end{tabular}

Data are presented as mean \pm standard deviation or number (\%).

ICU, intensive care unit.

Table 4. Analysis of Predictors of the Composite Outcome

\begin{tabular}{lrc}
\hline & Odds ratio $(\mathbf{9 5 \%}$ CI) & \multicolumn{1}{c}{$\boldsymbol{p}$-value } \\
\hline Age $(\mathrm{yr})$ & $0.518(0.231-1.162)$ & 0.109 \\
Gender & $0.656(0.265-1.624)$ & 0.361 \\
Bleeding etiology & $8.8(2.43-31.872)$ & $<0.001$ \\
Glasgow-Blatchford score & $1.486(0.632-3.498)$ & 0.363 \\
Endoscopy timing & $0.261(0.113-0.602)$ & 0.001 \\
Length of stay & $1.663(0.754-3.669)$ & 0.206 \\
\hline
\end{tabular}

CI, confidence interval.

Table 5. Analysis of Predictors of the Composite Outcome in Lower Risk Patients

\begin{tabular}{lcc}
\hline & Odds ratio $(95 \% \mathrm{CI})$ & $\boldsymbol{p}$-value \\
\hline Age $(\mathrm{yr})$ & $0.502(0.194-1.302)$ & 0.155 \\
Gender & $0.867(0.268-2.798)$ & 0.811 \\
Bleeding etiology & $10.568(2.191-50.967)$ & 0.001 \\
Endoscopy timing & $0.273(0.100-0.743)$ & 0.01 \\
Length of stay & $1.558(0.604-4.017)$ & 0.358 \\
\hline
\end{tabular}

$\mathrm{CI}$, confidence interval.

by bleeding severity and included both variceal and nonvariceal bleeding patients. Our stratified analysis revealed that the timing of endoscopy was not an important predictor of the composite outcome in high-risk groups or patients with variceal bleeding.

Our study had limitations mainly because it was retrospective, and we relied on medical records to measure the data and
Table 6. Analysis of Predictors of the Composite Outcome in Higher Risk Patients

\begin{tabular}{lcc}
\hline & Odds ratio $(\mathbf{9 5 \%}$ CI) & $\boldsymbol{p}$-value \\
\hline Age (yr) & $0.212(0.022-2.032)$ & 0.212 \\
Gender & $0.333(0.073-1.521)$ & 0.250 \\
Bleeding etiology & $5.923(0.628-55.853)$ & 0.204 \\
Endoscopy timing & $0.230(0.052-1.100)$ & 0.130 \\
Length of stay & $1.857(0.432-7.978)$ & 0.473 \\
\hline
\end{tabular}

CI, confidence interval.

Table 7. Analysis of Predictors of the Composite Outcome in Nonvariceal Bleeding Patients

\begin{tabular}{lcc}
\hline & Odds ratio $(95 \%$ CI) & p-value \\
\hline Age (yr) & $0.846(0.331-2.164)$ & 0.727 \\
Gender & $1.146(0.431-3.048)$ & 0.785 \\
Endoscopy timing & $0.260(0.098-0.687)$ & 0.006 \\
Length of stay & $1.467(0.595-3.617)$ & 0.405 \\
\hline
\end{tabular}

CI, confidence interval.

Table 8. Analysis of Predictors of the Composite Outcome in Variceal Bleeding Patients

\begin{tabular}{lcc}
\hline & Odds ratio (95\% CI) & $\boldsymbol{p}$-value \\
\hline Age (yr) & $0.233(0.018-3.026)$ & 0.530 \\
Gender & $0.095(0.004-2.175)$ & 0.230 \\
Endoscopy timing & $0.188(0.014-2.468)$ & 0.231 \\
Length of stay & $1.071(0.083-13.896)$ & 1.0 \\
\hline
\end{tabular}

CI, confidence interval. 
outcomes of interest. Furthermore, the timing of endoscopy was left to the discretion of the treating physician. Therefore, it could be speculated that patients with severe bleeding (and therefore at higher risk of rebleeding and mortality), preferentially underwent very early endoscopy, while those who were relatively hemodynamically stable preferentially underwent early endoscopy. Hence, it would be expected that the very early endoscopy group had worse outcomes. However, as shown in Table 2, clinical and laboratorial characteristics (such as GBS, hemoglobin, platelet count, International Normalized Ratio, blood pressure, heart rate, mental status, and comorbidities) were not significantly different in the very early versus early group, which suggested a similar clinical severity between the two groups.

In conclusion, we found that timing of endoscopy was an important predictor of poorer outcomes in nonvariceal UGIB, with very early endoscopy ( $\leq 12$ hours) associated with a higher chance of reaching a composite clinical outcome than early endoscopy (12-24 hours). Low-risk groups and patients with nonvariceal bleeding had poorer outcomes with very early endoscopy, whereas timing of endoscopy was not an important predictor in high-risk groups or patients with variceal bleeding. As previously stated, we still believe that additional data and research are required to justify very early endoscopy in patients with bleeding of non-variceal etiologies, with tailored studies further defining subgroups that may benefit most from earlier intervention. ${ }^{4}$ This is still a matter of debate and a controversial topic. Previous studies have led to different conclusions, so further prospective research is required to clarify the importance of prognostic scores for acute UGIB and the ideal timing of endoscopy.

\section{Conflicts of Interest}

The authors have no financial conflicts of interest.

\section{Author Contributions} Costa
Data Curation: GA

Formal analysis: Tiago Dias Domingues

Supervision: Jorge Reis

Writing-original draft: GA

Writing-review\&editing: GA, Luís Carvalho Lourenço

\section{REFERENCES}

1. Gralnek IM, Dumonceau JM, Kuipers EJ, et al. Diagnosis and management of nonvariceal upper gastrointestinal hemorrhage: European Society of Gastrointestinal Endoscopy (ESGE) guideline. Endoscopy 2015;47:a1-a46.

2. Hwang JH, Shergill AK, Acosta RD, et al. The role of endoscopy in the management of variceal hemorrhage. Gastrointest Endosc 2014;80:221227.

3. Garcia-Tsao G, Sanyal AJ, Grace ND, Carey WD. Prevention and management of gastroesophageal varices and variceal hemorrhage in cirrhosis. Am J Gastroenterol 2007;102:2086-2102.

4. Barkun A. What is the ideal timing for endoscopy in acute upper gastrointestinal bleeding? Endosc Int Open 2017;5:E387-E388.

5. Schacher GM, Lesbros-Pantoflickova D, Ortner MA, Wasserfallen JB, Blum AL, Dorta G. Is early endoscopy in the emergency room beneficial in patients with bleeding peptic ulcer? A "fortuitously controlled" study. Endoscopy 2005;37:324-328.

6. Bjorkman DJ, Zaman A, Fennerty MB, Lieberman D, Disario JA, Guest-Warnick G. Urgent vs. elective endoscopy for acute non-variceal upper-GI bleeding: an effectiveness study. Gastrointest Endosc 2004;60:1-8.

7. Tai CM, Huang SP, Wang HP, et al. High-risk ED patients with nonvariceal upper gastrointestinal hemorrhage undergoing emergency or urgent endoscopy: a retrospective analysis. Am J Emerg Med 2007;25:273278.

8. Lim LG, Ho KY, Chan YH, et al. Urgent endoscopy is associated with lower mortality in high-risk but not low-risk nonvariceal upper gastrointestinal bleeding. Endoscopy 2011;43:300-306.

9. Kumar NL, Cohen AJ, Nayor J, Claggett BL, Saltzman JR. Timing of upper endoscopy influences outcomes in patients with acute nonvariceal upper GI bleeding. Gastrointest Endosc 2017;85:945-952.e1.

10. Shih PC, Liu SJ, Li ST, Chiu AC, Wang PC, Liu LY. Weekend effect in upper gastrointestinal bleeding: a systematic review and meta-analysis. PeerJ 2018;6:e4248.

11. de Groot NL, Bosman JH, Siersema PD, van Oijen MG, Bredenoord AJ. Admission time is associated with outcome of upper gastrointestinal bleeding: results of a multicentre prospective cohort study. Aliment Pharmacol Ther 2012;36:477-484. 